\title{
NOUVELLe
}

\section{La protéinase 3}

\section{Signal de danger et agent double dans les vascularites}

Véronique Witko-Sarsat ${ }^{1}$, Sylvain Perruche ${ }^{2}$

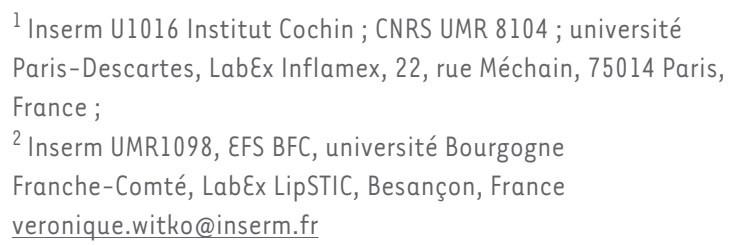
Paris-Descartes, LabEx Inflamex, 22, rue Méchain, 75014 Paris, France ;

${ }^{2}$ Inserm UMR1098, EFS BFC, université Bourgogne veronique.witko@inserm.fr

\section{Un défaut de résolution} de l'inflammation dans les vascularites auto-immunes : les polynucléaires neutrophiles sont les premiers suspects La granulomatose avec polyangéite (GPA), appelée autrefois maladie de Wegener, est une vascularite (inflammation de la paroi des vaisseaux) nécrosante caractérisée par une atteinte pulmonaire et rénale. C'est une pathologie grave et mortelle en l'absence de traitement [1]. Les patients atteints de cette maladie possèdent des auto-anticorps dirigés contre les polynucléaires neutrophiles et plus particulièrement contre la protéinase 3 (PR3), une protéase antibiotique localisée dans les granules de ces cellules. La GPA fait donc partie des vascularites nécrosantes touchant les petits vaisseaux et associées aux anticorps anti-cytoplasme de neutrophiles (ANCA). Son diagnostic se fait sur la base de la présence d'ANCA anti-PR3, considérés comme des biomarqueurs de cette maladie.

Les neutrophiles jouent un rôle clé dans cette vascularite puisqu'ils sont à la fois la cible de l'auto-immunité et les acteurs de la destruction de l'endothélium vasculaire [2]. Ils sont parmi les premières cellules immunitaires recrutées au site inflammatoire à la suite d'un dommage tissulaire ou d'une infection. Ils ont un rôle capital pour la défense contre les pathogènes, mais ils peuvent aussi avoir un effet délétère sur les tissus de l'hôte lorsqu'ils persistent au site de l'inflammation. Ils sont en effet doués de propriétés lytiques, en particulier grâce à leur capacité à générer des espèces réactives de l'oxygène (ROS) et à libérer des protéases capables de dégrader les tissus de l'hôte. Cependant, il est rassurant de constater que les neutrophiles sont capables d'enclencher leur mort programmée, ou apoptose, avant d'entrer dans cette phase délétère. L'apoptose des neutrophiles est une étape permettant l'initiation de la phase dite résolutive de la réponse inflammatoire. Leur élimination par les macrophages induit la sécrétion, dans ce microenvironnement, de facteurs prorésolutifs permettant l'arrêt de l'inflammation et l'initiation de la réparation tissulaire. Ces facteurs pro-résolutifs vont notamment limiter le recrutement d'autres leucocytes, optimiser l'élimination des cellules apoptotiques, et permettre une polarisation de la réponse immune vers un état de tolérance [3]. Il devient alors évident qu'une perturbation de ce mécanisme de résolution de la réponse inflammatoire, basé sur I'élimination des neutrophiles apoptotiques, pourrait favoriser le développement de pathologies inflammatoires auto-immunes.

Quand la protéinase 3 , cible des auto-anticorps, exprimée au cours de l'apoptose devient un signal de danger pour les macrophages Nous avons découvert qu'un scénario de ce type pourrait se dérouler dans la GPA [4]. En effet, nous avons montré que la protéinase 3 peut être exprimée à la membrane d'un neutrophile apoptotique [5], et en particulier sur les neutrophiles issus de patients atteints de cette maladie [6]. En utilisant des cellules myéloïdes transfectées avec la protéinase 3 , nous avons montré que la phagocytose de cellules en apoptose par des macrophages murins déclenche l'activation de ces derniers uniquement quand les cellules apoptotiques expriment la PR3 à leur surface. Cette activation se traduit par l'expression de l'oxyde nitrique synthase 2 (NOS2) et par la sécrétion de cytokines pro-inflammatoires en grandes quantités dont l'interleukine (IL)-12, I'IL-6, I'IL-1 $\beta$ et le facteur de nécrose tumorale (TNF)- $\alpha$. Ces macrophages activés libèrent également différentes chimiokines comme MCP-1 (monocyte chimoattractant protein 1 ), RANTES (regulated on activation, normal $T$ cell expressed and secreted), MIP-1-alpha (macrophage inflammatory protein I-alpha) et KC (keratinocyte chemoattractant) qui ont la capacité de recruter les monocytes, les lymphocytes, mais également les neutrophiles, permettant ainsi d'amplifier la réaction inflammatoire (Figure 1).

Grâce à l'utilisation de cellules transfectées avec une PR3 mutée sur un des acides aminés de la triade catalytique, et donc inactive, nous avons pu démontrer que l'activation du macrophage n'était plus observée lorsque les cellules apoptotiques exprimaient cette PR3 inactive, suggérant que l'activité enzymatique de la PR3 était indispensable à son activité inflammatoire. De la même façon, aucune activation du macrophage n'était observée lorsque la PR3 était mutée dans son domaine hydrophobe indispensable à son ancrage membranaire, démontrant l'importance de son expression à la surface cellulaire pour son activité pro-inflammatoire 


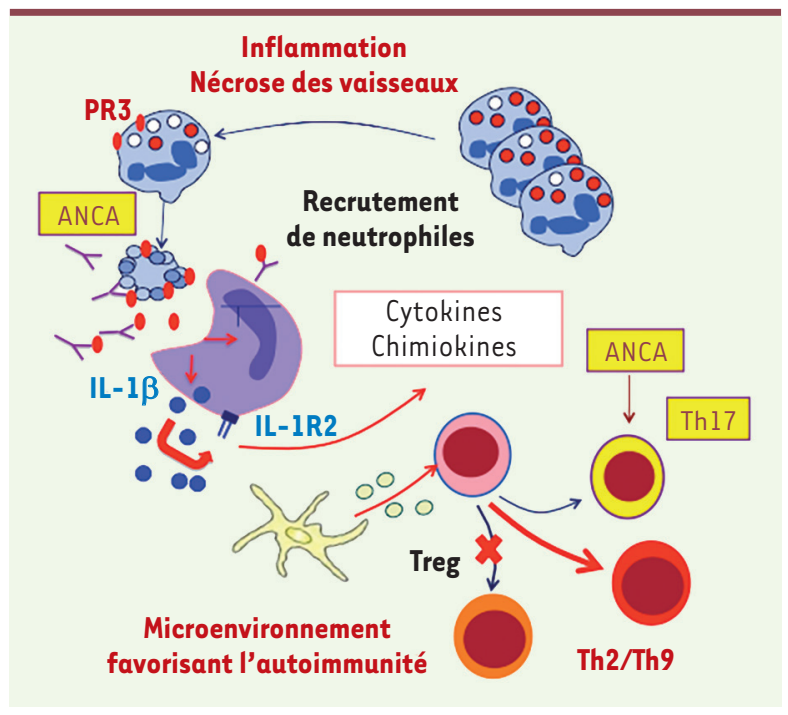

Figure 1. Le double jeu de la PR3 dans la GPA : signal de danger et autoantigène. Lors de la phagocytose, la PR3 exprimée à la surface des neutrophiles apoptotiques va activer les macrophages qui vont alors créer un microenvironnement riche en cytokines et chimiokines pro-inflammatoires, favorisant l'inflammation en particulier en activant la voie de I'IL-1 $\beta$. Ce microenvironnement va également promouvoir la stimulation des cellules dendritiques plasmacytoïdes et diminuer la génération de lymphocytes $T$ régulateurs, tout augmentant celle de lymphocytes Th9 et Th2, favorisant ainsi l'auto-immunité. De plus, la présence d'autoanticorps ANCA anti-PR3 va stimuler la génération de lymphocytes Th17, amplifiant le recrutement des neutrophiles. ANCA : anticorps anti-cytoplasme de neutrophiles; GPA : granulomatose avec polyangéite; IL-1 $\beta$ : interleukine-1-beta ; IL-IR : récepteur de l'interleukine 1 ; PR3 : protéinase 3 ; Th : lymphocyte T helper; Treg : lymphocyte T régulateur.
[6]. Ainsi, la PR3 exprimée sur la cellule apoptotique active le macrophage, comme un signal de danger, qui va générer un microenvironnement stimulant le système immunitaire.

La corruption des cellules dendritiques plasmacytoïdes favorise la mise en place d'une réponse auto-immune $\varepsilon n$ plus de leur rôle dans la réponse innée antivirale, les cellules dendritiques plasmacytoïdes ${ }^{1}$ jouent un rôle majeur dans la tolérance adaptative induite par les cellules apoptotiques [7]. Nous avons donc étudié leur devenir dans le microenvironnement généré par les macrophages éliminant des cellules apoptotiques exprimant la PR3. Les cellules dendritiques plasmacytoïdes, cultivées avec un surnageant de macrophages éliminant des cellules apoptotiques, induisent une polarisation des lymphocytes $T$ naïs vers un profil régulateurs (Treg). En revanche, des cellules dendritiques plasmacytoïdes traitées par le surnageant issu de macrophages éliminant des cellules apoptotiques $P R 3^{+}$favorisent une polarisation vers les profils pro-inflammatoires dits Th2 (lymphocyte T helper 2) et Th9 [13] $(\rightarrow)$.

$(\rightarrow)$ Voir la Synthèse de F. Vegran et al., $m / s n^{\circ} 4$, avril 2016, page 387

\footnotetext{
${ }^{1}$ Cellules dendritiques sécrétant de grandes quantités d'interféron de type I, fortement impliquées dans la réponse antivirale, mais aussi dans la réponse adaptative.
}

Ceci a été confirmé in vivo: des cellules dendritiques plasmacytoïdes issues de souris ayant reçu des cellules apoptotiques $\mathrm{PR}^{+}$, présentent des propriétés pro-Th9/Th2, aux dépens des Treg qui sont induits chez les souris ayant reçu des cellules apoptotiques PR3-. Nous avons de plus observé que si les autoanticorps anti-PR3, typiques de la GPA, étaient présents lors de l'injection de cellules apoptotiques $\mathrm{PR}^{+}$, les cellules dendritiques plasmacytoïdes favorisaient alors l'émergence de lymphocytes inflammatoires Th17 $[8,9](\rightarrow)$.

De façon intéressante, nous avons retrouvé ces populations lymphocytaires pro-inflam-

$\rightarrow$ Voir la Synthèse de S. Leung-TheungLong et $S$. Guerder, $m / s n^{\circ} 11$, novembre 2008, page 972 matoires (Th9/Th2 et Th17) chez les patients atteints de GPA. Enfin, la présence de macrophages au contact de cellules dendritiques plasmacytoïdes et de neutrophiles, au sein des granulomes inflammatoires présents au niveau pulmonaire (Figure 2), nous suggère fortement que le dialogue cellulaire et le scénario que nous avons décrit pourraient se dérouler chez les patients (Figure 1).

Vers de nouvelles perspectives thérapeutiques ciblant les voies activées par PR3 ?

Pour la première fois, cette activation du macrophage par la PR3 présente à la surface de neutrophiles apoptotiques, dépendant de la voie de l'interleukine-1, met en évidence une composante autoinflammatoire dans la granulomatose avec polyangéite [10], ouvrant ainsi la voie à de nouvelles pistes thérapeutiques. Les différentes molécules disponibles et capables d'inhiber la production de I'IL-1 $\beta$, molécules déjà utilisées dans d'autres maladies comme la goutte et la fièvre méditerranéenne familiale? pourraient compléter le traitement standard actuel composé de corticostéroïdes et d'immunosuppresseurs, et plus récemment d'anti-CD20 ciblant les lymphocytes B [11].

Les cellules dendritiques plasmacytoïdes peuvent être impliquées dans des mécanismes pro-inflammatoires déjà documentés dans la polyarthrite rhumatoïde ou encore la maladie de $\mathrm{Crohn}^{3}$, au cours desquelles elles favorisent la polarisation des lymphocytes pathogéniques [12]. À l'opposé, les cellules dendritiques plasmacytoïdes ont un effet anti-inflammatoire bien documenté dans les tumeurs, dont le microenvironnement riche en facteurs anti-inflammatoires va leur permettre d'entretenir les lymphocytes

\footnotetext{
${ }^{2}$ La fièvre méditerranéenne familiale ou maladie périodique, est une maladie auto-inflammatoire caractérisée par des crises fébriles brèves récurrentes et des inflammations des membranes séreuses provoquant des douleurs abdominales, à la poitrine, arthralgies et myalgies.

${ }^{3}$ Maladie inflammatoire du tube digestif.
} 


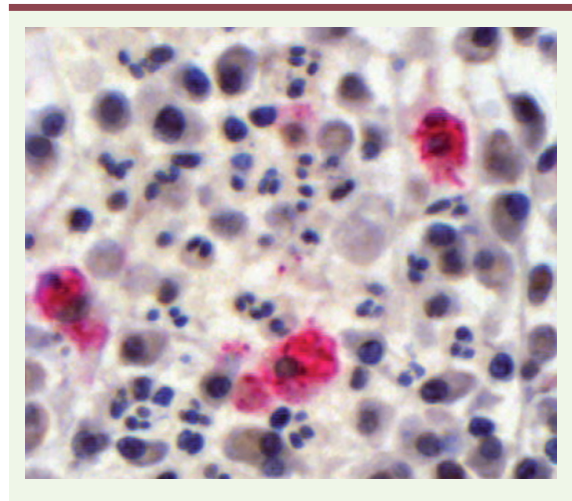

Figure 2. Analyse immunohistochimique des différentes cellules inflammatoires dans un granulome pulmonaire chez un patient atteint de GPA. Grâce à des marquages spécifiques, les macrophages apparaissent en rose magenta $\left(C D 68^{+}\right)$et les cellules dendritiques plasmacytoïdes en marron $\left(\operatorname{CD} 123^{+}\right)$. Les neutrophiles sont reconnaissables par leur noyau polylobé, coloré en bleu foncé, qui apparaît sous forme de trois «boules». Tous ces types cellulaires sont donc présents au sein des lésions inflammatoires typiques de la GPA. GPA : granulomatose avec polyangéite (Photos: Antje Müller, Université de Lübeck, Allemagne).

régulateurs. La découverte du rôle clé des cellules dendritiques plasmacytoïdes dans la dérégulation immunitaire associée à la GPA permet d'envisager des stratégies thérapeutiques basées sur la reprogrammation de ces cellules [12]. Enfin, la modulation de l'activité ou de la localisation de la PR3 par des agents pharmacologiques sont autant d'approches pertinentes et innovantes pour un traitement adapté à cette maladie. $\diamond$ Proteinase 3: a new danger signal and a double agent in vasculitis

\section{LIENS D'INTÉRÊT}

Les auteurs déclarent n'avoir aucun lien d'intérêt concernant les données publiées dans cet article.

\section{RÉFÉRENCES}

1. Jennette JC, Falk RJ. Pathogenesis of antineutrophil cytoplasmic autoantibody-mediated disease. Nat Rev Rheumatol 2014 ; 10 : 463-73.

2. Witko-Sarsat V. Neutrophils in ANCA-associated vasculitis: still under investigation. Presse Med 2013; $42: 595-7$.

3. Poon IK, Lucas CD, Rossi AG, Ravichandran KS. Apoptotic cell clearance: basic biology and therapeutic potential. Nat Rev Immunol 2014 ; 14 : 166-80.
4. Gabillet J, Millet A, Pederzoli-Ribeil M, et al. Proteinase 3 , the autoantigen in granulomatosis with polyangiitis, associates with calreticulin on apoptotic neutrophils, impairs macrophage phagocytosis, and promotes inflammation. J Immunol 2012 ; 189 : 2574-83.

5. Kantari C, Pederzoli-Ribeil M, Amir-Moazami 0, et al. Proteinase 3 , the Wegener autoantigen, is externalized during neutrophil apoptosis: evidence for a functional association with phospholipid scramblase 1 and interference with macrophage phagocytosis. Blood 2007 ; 110 : 4086-95.

6. Millet A, Martin KR, Bonnefoy F, et al. Proteinase 3 on apoptotic cells disrupts immune silencing in autoimmune vasculitis. J Clin Invest 2015 ; 125 : 4107-21.

7. Bonnefoy F, Perruche $S$, Couturier M, et al. Plasmacytoid dendritic cells play a major role in apoptotic leukocyte-induced immune modulation. J Immunol 2011 ; 186 : 5696-705.

8. Bonnefoy F, Couturier M, Clauzon A, et al. TGF-betaexposed plasmacytoid dendritic cells participate in Th17 commitment. J Immunol 2011 ; 186: 6157-64.

9. Leung-Theung-Long S, Guerder S. Les cellules Th17: Une nouvelle population de cellules T CD4 effectrices pro-inflammatoires. Med Sci (Paris) 2008 ; 24 : 972-6.

10. Grateau G, Hentgen V, Stojanovic KS, et al. How should we approach classification of autoinflammatory diseases? Nat Rev Rheumatol 2013 ; $9: 624-9$.

11. Millet A, Pederzoli-Ribeil M, Guillevin L, et al. Antineutrophil cytoplasmic antibody-associated vasculitides: is it time to split up the group? Ann Rheum Dis 2013; 72 : 1273-9.

12. Saas $P$, Kaminski $S$, Perruche $S$. Prospects of apoptotic cell-based therapies for transplantation and inflammatory diseases. Immunotherapy 2013; 10 : 1055-73.

13. Vegran F, Martin F, Apetoh L, Ghiringhelli F. Les lymphocytes Th9 : une nouvelle population de lymphocytes T auxiliaires. Med Sci (Paris) 2016 $32: 387-93$.
NOUVELLE

Le récepteur neural
des cestrogènes bêta
Un nouvel acteur dans la maturation
pubertaire femelle
Lydie Naulél-3, Sakina Mhaouty-Kodja

> La puberté est une période critique du développement qui permet la transition entre l'enfance et l'adolescence. Elle se caractérise par l'augmentation des niveaux d'hormones gonadiques, l'apparition des caractères sexuels secondaires et l'acquisition de la capacité à se reproduire. Le déclenchement de la puberté est régulé par de nombreux facteurs. Des études épidémiologiques ont montré que 50 à $80 \%$ des variations de l'âge de la puberté sont déterminés par des facteurs génétiques [1]. II est également établi que des facteurs environnementaux, comme les conditions socio-économiques, la nutrition, le stress, ou encore l'exposition à des perturbateurs endocriniens, jouent un rôle important dans le déclenchement de la puberté [2]. Aujourd'hui, l'observa-
${ }^{1}$ Neuroscience Paris Seine, Inserm, UMR S1130, Université Pierre et Marie Curie, 7, quai Saint-Bernard, 75005 Paris, France; ${ }^{2}$ CNRS, UMR 8246, Université Pierre et Marie Curie, Paris, France ;

${ }^{3}$ Sorbonne Universités, Université Pierre et Marie Curie UM CR18, université Paris 06, France.

lydie.naule@yahoo.fr sakina.mhaouty-kodja@upmc.fr

tion d'une avancée progressive de l'âge de la puberté chez les filles à travers le monde, soulève une forte inquiétude. Les causes de cette avancée ne sont pas clairement établies mais l'augmentation de l'obésité infantile et l'exposition grandissante à des polluants environnementaux y contribueraient fortement. Du fait des effets délétères que peut avoir une perturbation de l'âge de la puberté 